\title{
Medical Telemetry System for Monitoring and Localization of Patients - Functional Model and Algorithms for Biosignals Processing
}

\author{
Marek Kurzyński, Przemysław Ryba, Marcin Markowski, and Michał Woźniak
}

\begin{abstract}
The article presents the concept of hospital telemetric system. The goal of the project is a model of early warning systems for patients outside intensive care wards. Proposed system is based on constant telemetric monitoring using objective physiological parameters. Using low-distance sensor network which covers body of a patient, so-called BAN (Body Area Network) is the main innovation of the project. Some preliminary results of ECG analysis and interpretation modules and units of proposed system will be presented.
\end{abstract}

Keywords-Telemedicine, mobile health system, patient monitoring, remote diagnostic.

\section{INTRODUCTION}

$\mathbf{S}$ IGNIFICANT and actual problem of health protection systems is to ensure suitable safety during taking care of patient. One element patients threats for are mistakes in proceeding way: delay in identification of health danger or delayed or incorrect therapeutic intervention.

The report "To Err is Human" worked out in the USA, states that due to clinical mistakes which were possible to be avoided each year dies $44000 \div 98000$ patients [1]. The analysis of clinical mistakes occurred during hospital treatment showed that they are to the higher degree result of incorrect structure and hospital care system organization than individual medical mistake.

Full safety is assured for the sick who are cured in intensive care hospital ward, however maintaining places in these wards is very expensive. Beds on intensive care wards make up in the USA $15 \%$ of all hospital beds, in Western Europe $3 \div 8 \%$, and in Poland $1 \%$ [2].

The patient's safety improvement in hospitals is to introduce organizational solutions to the existing system which will let to faster identification of health danger and efficient therapeutic intervention carried out by personnel trained in intensive care. Such solution was implemented in recent years by a couple of developed countries, at the earliest by Australia and the United Kingdom. The Australian system was called MET (Medical Emergency Team), and English ICORS (Intensive Care Outreach Services) [3]. They work according to the similar rules. Personnel of hospital wards using clinical criteria of patient condition evaluation based on punctual clinical scales (EWS - early warning score) identifies health danger and informs

M. Kurzyński, P. Ryba, M. Markowski, and M. Woźniak are with Department of Systems and Computer Networks, Wrocław University of Technology, Wyb. Wyspiańskiego 27, Wrocław, Poland (e-mails: marek.kurzynski@pwr.wroc.pl, przemyslaw.ryba@pwr.wroc.pl, marcin.markowski@pwr.wroc.pl,michal.wozniak@pwr.wroc.pl). according to defined system personnel of intensive care ward. The efficiency of MET and ICORS systems was assessed and measured. It was proved that after MET implementation the risk of hospital heart action stop decreased by $66 \%$ and general hospital mortality went down by $26 \%$ [4]. Among patients cured by surgical procedure the death risk lowered by $37 \%$.

Implementation of the system is justified on these wards where stays one of the following groups of increased risk patients:

1) Patients admitted to hospital in emergency cases subjected to routine hospital procedures. The most dangerous complication in this group is sudden stop of heart action.

2) Sick persons with general infection symptoms, by whom early detection of organism functions disorder should lead to reduction of serious general infection frequency and septic shock - clinical syndromes which are characterized by very high treatment costs and mortality rate.

3) Patients after operation who are specifically endangered population. The analysis of deaths among the sick in this period shows that $60 \%$ of them happen a couple days after operation when patients stay on hospital ward without intensive care.

4) Sick persons after longer stay on intensive care ward who are moved to mother hospital ward and still require intensified supervision and more intensive nursery and therapeutic procedures.

Another field of practical application of the system is remote monitoring of persons located outside the hospital, like monitoring patients at home [5], sportsmen monitoring [6], emergency medicine [7].

Convalescent could recover at home and remote monitoring system should inform doctor at hospital about patient condition.

Such solution brings significant savings for hospital and it is much more comfortable for patients, who do not stress while recovering at their homes. Nowadays European societies become older and older. It is obvious that the medical care demands and monitoring cost of older people condition will grow in next years. It is impossible to guarantee the proper monitoring of the whole aforementioned population in traditional way. Often they live alone and they are exposed to risk of their lives and health - by accidents, collapses, heard attacks, and other. Implementing remote monitoring system specialized for those groups of people seem to be the cheapest and most efficient way of their protection. 
Remote health monitoring system is dedicated to the following groups of patients:

- Patients at home, i.e. people who usually stay at home and seldom leave, like convalescents after hard illness, patients during rehabilitation, bed-ridden patients, who do not require hospital care, patients physically handicapped, older people with limited movement capabilities.

- Patients in move - working, travelling, practicing sport, leading usual live. Sportsmen, patients with chronic diseases, patients with high risk of some illness belong to this group. They need continuous monitoring of chosen parameters and additionally a localizing system in case of rapid emergences.

\section{TElemedicine Systems}

Among different services realized by telemedicine systems, a key role plays remote monitoring of chosen biosignals. Studies in this field - numerous also in stationary technology - gained additional impulse in the moment when technical possibilities of building up mobile systems has emerged. So called m-health [3] it is to large degree mobile monitoring systems using wireless techniques, which ensure patient possible maximum comfort by keeping complete ergonomics.

On the basis of analysis of the newest studies in remote monitoring systems we could define development trends and describe exemplary practical solutions, both in the field of utilized concepts and realized range of medical services [8][11]. It evidently results from them that in application field, projected mobile monitoring systems [12] concern remote care of patient staying at home and remote care of hospitalized one.

The above requirements imposed the following technical solutions:

1) Comfortable system of sensors, e.g. multifunctional wrist sensor system or more technologically advanced concepts, so called wearing sensors [13]. The example may be Georgia Tech Wearable Motherboard [14], it means "intelligent" fabric with appropriate plaited sensors, energy source and electronic network and similar solutions in the form of so called "intelligent clothes": VinsurespTM (RBI, France), Wealthy shirt or LifeshirtTM (Vivometrics, USA)

2) Commonly utilization of wireless individual computer network (WBAN) [15] for comfortable acquisition of chosen signals, working most often in IEEE 801.15.1 standard (BlueTooth) [16] with devices PDA class performing network server role.

3) Utilization of mobile network to communication with proper telemedicine services center and next with doctor or hospital [17]. Network server communicates with device equipped with GSM phone and GPS receiver. Patient monitoring data are sent through the mobile network to the hospital network and then to the monitoring center. In case of patients staying at home (belong to group 5, described in section 1) also home LAN and Internet connection or stationary PSTN telephony network may be utilized.
4) Common utilization of GPS or cellular-based patient localizing system for patients in motion (belong to group 6 , described in section 1).

More modest proposals of monitoring systems are designed for hospital usage. As it results from analysis of actual literature reports [18]-[20], there is actually no efficiently operating system for hospitals usage based on physiological parameters analysis and the first trials of working out similar systems are only in project phase.

Telemetric systems offered by some companies, although they can also be utilized in hospital practice, have no characteristics of holistic solution, but enable only tracking some chosen parameters. The example can be Guardian RT system worked out by Medtronic company enabling stable monitoring of glucose level, or telemetric solutions built up by Philips letting to follow the parameters coming from oxymeter or mobile electrocardiograph.

\section{Project GoAls}

The aim of the project is to work out and examine the prototypical implementation of hospital system of early detecting patient condition danger based on constant telemetric monitoring system utilizing objective physiological parameters (e.g. body temperature, heart beat, blood pressure, breaths frequency, blood saturation and electrocardiography).

Most important features of the systems are:

1) Mobility, it means enabling patients using system services and supervising them medical personnel to free movement in hospital premises.

2) Non-arduousness meaning high "transparency" of the system for sick persons and her/his routine daily activities (meals, personal hygiene, sleep, etc.).

3) Scalability, it is possibility of simultaneously benefiting system services by unlimited number of patients and personnel.

4) Openness, which enable adding to the system in easy way new monitored signals and enrichment of monitoring scenario by new clinical situations.

Proposed system makes possible to realize the following goals:

1) Constant, remote monitoring of selected parameters and physiological signals of defined group of patients and their visualization on central station.

2) Monitoring condition of chosen patient through PC or PDA by the doctor.

3) Carrying out on-line analysis of incoming data from patient sensors according to the procedures worked out by doctors and defined clinical criteria in order to evaluate patient condition and detecting life danger situations which require immediate intervention.

4) Utilizing data for further analysis in order to asses taken actions and work out therapeutic proceeding way standards and algorithms letting to forecast patient condition.

5) Defining synthetic indicators from monitoring process to simplify creation of medical documentation.

6) Movement monitoring of 'patients in move' in order to be enable to localize patient in case of emergency. 


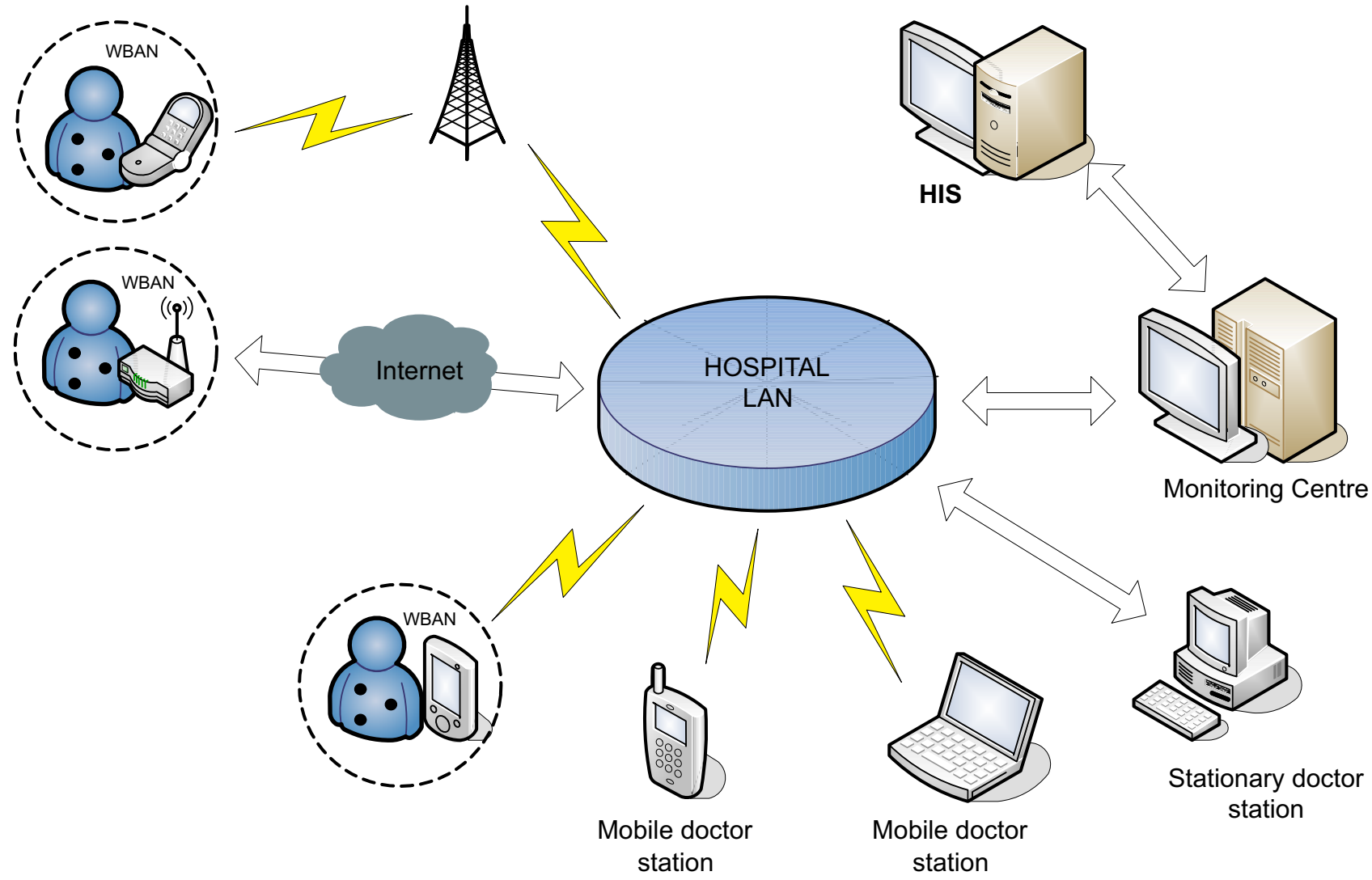

Fig. 1. Structure of patients monitoring system.

Targets of the system presented above will be realized through fulfilling appropriate functional requirements imposed on hardware and software of the system components, i.e. patient monitoring station, physician workstation and central monitoring station collecting and visualizing data about all supervised patients.

Final outcome of the project will be result of technical and functional studies on the hospital early warning system prototype. Obvious material element after project finalization will be built up system equipment and structural documentation of the hardware and software.

System prototype will be subjected to preliminary evaluation with regard to effectiveness and usefulness and hospital practice.

\section{System Model AND REQUIREMENTS}

The basic concept of the monitoring system structure is presented in the Figure 1. Wireless body area network (WBAN) is composed of sensors and mobile patient station (MPS) also called LPU (Local Processing Unit). WBAN works in the client-server mode. Sensors works under control of LPU, LPU collects data and communicates with monitoring centre at hospital.

For patients 'in move' LPU function is fulfilled by smart phone equipped with GPS receiver. WBAN communicates with monitoring centre over cellular network and then over hospital LAN (local area network). GPS data, necessary for patient localization, are also sent to central monitoring system at hospital.

For patients staying at home the role of LPU is fulfilled by a PDA device. PDA communicates with home wireless LAN, connected to Internet. For data confidentiality communication between home LAN and hospital LAN should be realized via VPN connection. Utilization of Internet allows to reduce communication costs.

Patients at hospital are equipped with PDA device, which collects data from sensors and provides wireless communication with hospital LAN.

Hospital monitoring centre, connected to the hospital information system (HIS) with HL7 standard, collects and analysis data from WBANs and alarms in case of emergencies. Whenever doctor reaction is necessary, monitoring centre requests doctor via hospital LAN. Doctors may use mobile wireless PDA device, laptop or stationary computer. Monitoring centre with cooperation with HIS may also provide additional functions, like localizing the nearest doctors or medical emergency teams.

The main tasks of mobile patient station are: collecting data from sensors, monitoring of critical signals, alarming in case of emergency or system failure, communicating with central monitoring centre at hospital. 


\section{Preliminary RESUlts}

\section{A. ECG Signal Analysis and Interpretation}

The most important stage of ECG analysis consists in detection of fiducial points (onset and offset of $\mathrm{P}, \mathrm{QRS}$ and $T$ waves), because its quality has significant influence on the quality of the whole system. Usually, the detection of QRS complexes and $\mathrm{P}$ and $\mathrm{T}$ waves is composed of two-stages [21], [22]. First the so-called detection function is constructed, which has a smooth shape with local maxima for each QRS complex and $\mathrm{P}$ and $\mathrm{T}$ wave. Next, the location of QRS complexes and $\mathrm{P}$ and $\mathrm{T}$ waves on the time axis is estimated, applying some rules concerning the detection function.

In the analysis module of CARDIO system to the construction of detection function the energy measure of fuzziness method was applied, which has been reported as a powerful tool for ECG signal processing [23]. The energy measure of fuzziness is a method for quantitative assessment of fuzzy uncertainty in signals, which fulfils two postulates [24], [25]:

- For the constant signal containing no fuzzy uncertainty, the energy measure of fuzziness is equal to zero, which means that the information conveyed by such a signal is maximal.

- Higher dynamics of the changes of the original signal denotes the higher value of energy measure of fuzziness. It means that the amount of information is smaller for the variable signal than for the constant one.

As an example, an ECG signal MO1_004 (II lead) from data-base CSE is shown in Fig. 2. However Fig. 3 illustrates a course of energy measure of fuzziness for this signal. Since the course corresponds to a single peak of QRS complexes and furthermore, $\mathrm{P}$ and $\mathrm{Q}$ waves are represented by peaks with small amplitude, the presented course of energy measure of fuzziness may be used as a detection function.

As an result of the performance of analysis module, we receive the following ECG parameters:

- Amplitudes of P, Q, R, S, T waves, amplitude and slope of ST interval (for each lead).

- Duration of Q, R, S waves (for each lead), duration of $\mathrm{P}$ wave, PQ segment, QT interval and QRS complex (absolute values).

- Number of P waves, localization of PM spikes (if any), flags for $\mathrm{F} / \mathrm{f}$ waves detection.

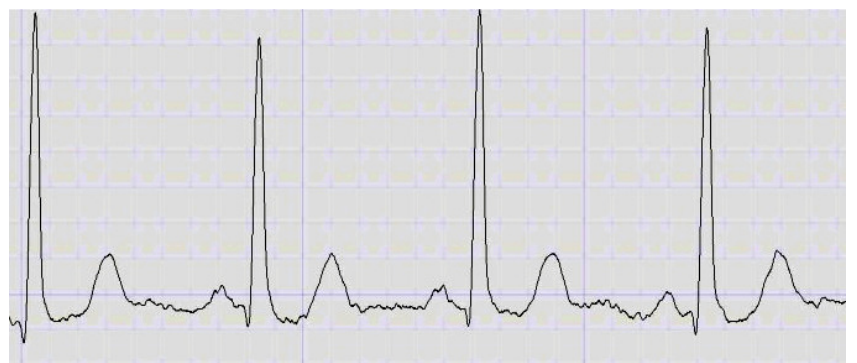

Fig. 2. ECG signal MO1_004 from CSE data base.
TABLE I

The Available ECG Disorders (ACCORding to AHA Systematics [26] AND NOVACOdE CLASSIFICATION SYSTEM [27])

NORMAL TRACING: Normal ECG.

TECHNICAL PROBLEMS: Leads misplaced, Artifacts.

SINUS NODE RHYTHMS AND ARRHYTHMIAS: Sinus rhythm,

Sinus tachycardia ( $>100$ beats per minute), Sinus bradycardia

$(<50$ beats per minute), Sinus arrhythmia,Sinus arrest or pause,

Sino-atrial exit block.

OTHER SUPRAVENTRICULAR RHYTHMS: Atrial premature complexes, Ectopic atrial rhythm, Ectopic atrial tachycardia, Atrial fibrillation, Atrial flutter, Junctional premature complexes,

Junctional escape complexes or rhythm, Accelerated junctional rhythm, Junctional tachycardia, Supraventricular tachycardia.

VENTRICULAR ARRHYTHMIAS: Ventricular premature

complexes, Ventricular escape complexes or rhythm, Accelerated idioventricular rhythm, Ventricular tachycardia, Ventricular fibrillation. ATRIAL VENTRICULAR CONDUCTION: First-degree AV block,

Mobitz Type 1 second-degree AV block (Wenckebach), Mobitz Type 2 second-degree AV block, AV block or conduction ratio, 2:1, AV block, varying conduction ratio, $\mathrm{AV}$ dissociation.

INTRAVENTRICULAR CONDUCTION: Left bundle branch block,

Right bundle branch block (complete or incomplete), Left anterior fascicular block, Left posterior fascicular block, Wolff-Parkinson-White syndrome.

QRS AXIS AND VOLTAGE: Right axis deviation, Left axis

deviation, Indeterminate axis, Low voltage (less than $0.5 \mathrm{mV}$ total QRS amplitude in each extremity lead and less than $1.0 \mathrm{mV}$ in each

Precordial lead).

CHAMBER HYPERTROPHY OR ENLARGEMENT: Left ventricular hypertrophy (with or without ST-T abnormality), Right ventricular hypertrophy (with or without secondary ST-T abnormality). MYOCARDIAL INFARCTIONS AND/OR ISCHEMIC

ABNORMALITIES: Q wave MI, Isolated ischemic abnormalities, Isolated minor Q and ST-T abnormalities.

PACEMAKER: Atrial-paced rhythm, Ventricular-paced rhythm,

Atrial-sensed ventricular-paced rhythm, Failure of appropriate capture,

Failure of appropriate inhibition, Failure of appropriate inhibition,

Failure of appropriate pacemaker firing.

Parameters of ECG signal are the basis of ECG interpretation. The set of ECG disorders supported by the interpretation module is presented in the Table 1 .

\section{B. Modeling and Traffic Simulation of the Sensors BAN}

The phase of modeling and simulation of the Wireless Body Area Network activity is important from practical point of view. During this phase the initial analysis of system operation is provided and some improper assumption or designing mistakes may be detected. On the simulation stage, there is a possibility to solve some problems cheaper and faster

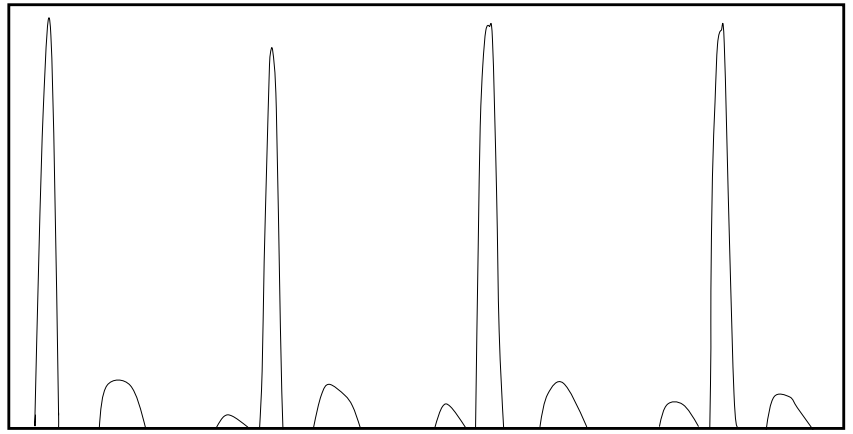

Fig. 3. Energy measure of fuzziness for signal from Fig. 2. 
than performing the multiple tests during prototype evaluating. There are some known problems for which simulation of the Body Area Network operation should find solutions and answers.

One of the main problems of wireless sensors networks is optimization of power efficiency [28]. An important quality indicator of the designed network is the maximal operation time of sensors and mobile patient station (PDA, MDA or smartphone) using battery power supply. Since mobile patient station is equipped with much larger battery than sensors, the major issue in WBAN designs is how to save power of wireless sensors. In the considered problem, the main reason for power effort is wireless communication between elements of BAN network. It is worth to notice, than devices communicated with Bluetooth or 802.11 standard utilize much more power than ZigBee standard solutions, but most available sensors and PDA devices are equipped with Bluetooth modules. Beside that, taking ZigBee technology into account seems to be reasonable, because this technology is strongly promoted in healthcare applications and is expected to gain market share in the future. Surveying the literature, results of experiments concerning power efficiency in wireless sensor networks show, that maximal operation time of such network depends on few easy to predicts determinants [29]. Those determinants may be predicted and optimized during designing the sensor network and remote monitoring system. The most important of them are: network topology [30], [31] (power consumption spent on packet forwarding in single-hop star topology can be omitted) and communication manner (including communication protocols [32], [33]), number of devices in the network, distance between devices, amount of transmitted data, the incidence of transmissions (high power effort is observed especially while changing mode from hibernation to transmitting), presence of other wireless networks resulting in overhearing (nodes waste energy to receive packets not belonging to them, which will be dropped) and collisions (due to transmission failures extra power is consumed on retransmissions). In order to make the optimal choice of network parameters wide simulation experiments, including different topologies and sensors deployment, will be conducted.

Another important BAN designing aspects, in which simulations may be very helpful is determining the correctness and efficiency of a design. Simulation phase lets us to monitor the activity correctness of communication protocols and overall system behavior in different operating conditions. We anticipate, that during that phase of our project we will be able to detect possible problems, like the lack of compatibility between protocols, duplication of messages, unavailability of some sensors, different times demanded by sensors for changing from idle mode to transmission mode, etc. Tests provided with our monitoring system in simulator allow us to shorten verification time, automate experiments and perform more tests with more number of system states and communication scenarios.

In the health monitoring system reliability and availability are highly demanded. It is unacceptable that packet containing data from one of the sensors may not reach the patient station and then the hospital monitoring centre. Simulations of the
BAN network operating will let us try different possibilities of routing in wireless BAN environment (i.e. different network topologies) and choose the best solution.

Wireless body sensor networks have specific timing requirements [34]. Simulation experiments are helpful for correlating of protocols and transmission parameters in order to make them accurate for all devices (sensors and mobile patient station) connected to the BAN network. Also possibility and usefulness of cooperating of ZigBee and Bluetooth devices in one Body Area Network will be investigated.

\section{FINAL REMARKS}

Paper presented the main assumptions and preliminary results of selected works devoted to remote monitoring system. In the light of finished works, we should expect the following results of system implementation in hospital practice:

- Improvement of patient safety in hospital wards.

- Decreasing of mortality during stay in hospital caused by delayed or incorrect therapeutic intervention.

- Ensuring patient safety without need to increase number of very expensive treatment stands on intensive care ward and - in the future - resulting from shortening patients stay in hospital due to possibility of monitoring patient at home.

\section{REFERENCES}

[1] L. T. Kohn, To Err is Human: Building a Safer Health System. Washington DC: National Academy Press, 2000.

[2] P. McQuillan, "Confidential inquiry into quality of care before admission to intensive care," BMJ, vol. 316, pp. 1853-1858, 1998.

[3] "Going mobile: From eHealth to mHealth, a Daou systems white paper," April 2001.

[4] R. Bellomo, "A prospective before-and-after trial of a medical emergency team," The Medical Journal of Australia, vol. 173, pp. 257-263, 2003 .

[5] P. E. Ross, "Managing care through the air," IEEE Spectrum, vol. 41, no. 12 , pp. 26-31, 2004.

[6] E. Callaway, Wireless Sensor Networks: Architectures and Protocols. CRC Press, 2004.

[7] M. Miyazaki, "The future of e-health wired or not wired," in Science of Computer Programming, 2003.

[8] C. May, T. Finch, F. Mair, and M. Mort, "Towards a wireless patient: Chronic illness, scare care and technological innovation in the UK," Social Science \& Medicine, vol. 61, pp. 1485-1494, 2005.

[9] A. Dwivedi, "The efficacy of the m-health paradigm: incorporating technological, organizational and managerial perspectives," in International Conference on Telemedicine, Bari, 2003, pp. 15-32.

[10] R. Haux, "Health, information systems - past, present, future," International Journal of Medical Informatics, vol. 75, pp. 268-281, 2006.

[11] C. Pattichis and E. Kiriacou, "Wireless telemedicine systems: an overview," IEEE Antennas and Propagation Magazine, vol. 44, pp. 143 157, 2004.

[12] M. Biemer and J. Hampe, "A mobile medical monitoring system: Concept, design and deployment," in IEEE Conference on Mobile Business, 2005, pp. 37-42.

[13] S. Park and S. Jayaraman, "Enhancing the quality of life through wearable technology," in IEEE Engineering in Medicine and Biology Magazine, 2003, pp. 41-48.

[14] B. Firoozbakhsh and N. Sungmee, "Wireless communication of vital signs using the Georgia Tech wearable motherboard," in IEEE Engineering in Medicine and Biology Society Conference, 2000, pp. 1253-1256.

[15] Y. Lin and I. Jan, "A wireless PDA-based physiological monitoring system for patient transport," in IEEE Transactions on Information Technology in Biomedicine, vol. 8, 2004, pp. 439-445.

[16] M. Fadlee and B. Wood, "Bluetooth telemedicine processor for multichannel biomedical signal transmission via mobile cellular network," in IEEE Transactions on Information Technology in Biomedicine, vol. 9, 2005, pp. 35-42. 
[17] C. Salvador and M. Carrasco, "Airmed-cardio: A GSM and internet services-based system for out-of-hospital follow-up of cardiac patients," in IEEE Transactions on Information Technology in Biomedicine, vol. 9 , 2005, pp. 73-85.

[18] H. Marin, "Improving patient safety with technology," International Journal of Medical Informatics, vol. 73, pp. 543-546, 2004.

[19] N. Maglevaras and I. Chuovarda, "The citizen health system (CHS): modular medical contact center providing quality telemedicine services," in IEEE Transactions on Information Technology in Biomedicine, vol. 9 , 2005, pp. 353-361.

[20] E. Kafeza, D. Chiu, and C. Chueng, "Alerts in mobile healthcare applications: requirements and pilot study," in IEEE Transactions on Information Technology in Biomedicine, vol. 8, 2004, pp. 173-178.

[21] J. A. Milliken, H. Pipberger, and H. V. Pipberger, "The impact of an ECG computer analysis program on the cardiologist's interpretation. a cooperative study," Journal of Electrocardiology, vol. 16, no. 2, pp. 141$149,1983$.

[22] T. S. K. Endou, H. Miyahara, "Clinical usefulness of computer diagnosis in automated electrocardiography," Cardiology, vol. 66, no. 3, pp. 174 89, 1980.

[23] E. Czogała and J. Łęski, "Application of entropy and energy measures of fuzziness to processing of ECG signal," Fuzzy Sets and Systems, vol. 97, pp. 9-18, 1998.

[24] O. Pahlm and L. Sornmo, "Data processing of exercise ECG signal," in IEEE Transactions on Biomedical Engineering, vol. 34, 1987, pp. $158-165$.

[25] J. Knopfmacher, "On measures of fuzziness," Journal of Mathematical Analysis and Applications, vol. 49, pp. 529-534, 1975.

[26] A. H. Kadish, "ACC/AHA clinical competence on electrocardiography," Journal of American College of Cardiology, vol. 38, pp. 2091-2099, 2001.

[27] P. Rautaharju, "The novacode criteria for classification of ecg abnormalities and their clinically significant progression and regression," Journal of Electrocardiology, vol. 31, pp. 157-187, 1998.
[28] C. ShihHeng and H. Ching Yao, "Power model for wireless body area network," in Biomedical Circuits and Systems Conference, 2008.

[29] M. Sukor, S. H. S. Ariffin, N. Fisal, S. K. S. Yusof, and A. Abdallah, "Performance study of wireless body area network in medical environment," in First Asia International Conference on Modelling and Simulation, Kuala Lumpur, Malaysia, 2008, pp. 202-206.

[30] W. R. Heinzelman, A. Chandrakasan, and H. Balakrishnan, "Energy efficient communication protocol for wireless microsensor networks," in Hawaii International Conference System Sciences Maui, 2000, p. January.

[31] W. Ye, J. Heidemann, and D. Estrin, "An energy-efficient MAC protocol for wireless sensor networks," in Twenty-First Annual Joint Conference of the IEEE Computer and Communications Societies, 2002, pp. 15671576.

[32] B. Latre, B. Braem, I. Moerman, C. Blondia, E. Reusens, W. Joseph, and P. Demeester, "A low-delay protocol for multihop wireless body area networks," in Proceedings of the 4th Annual International Conference on Mobile and Ubiquitous Systems: Computing, Networking and Services, 2007, pp. 1-8.

[33] T. van Dam and K. Langendoen, "An adaptive energy-efficient MAC protocol for wireless sensor networks," in Proceedings of the 1st International Conference on Embedded Networked Sensor Systems, Los Angeles, California, USA, November 2003, pp. 171-180.

[34] Y. Liang, A. Roychoudhury, and T. Mitra, "Timing analysis of body area network application," in 7th International Workshop on Worst-Case Execution Time Analysis, 2007.

[35] D. Bright, "Outreach-a strategy for improving the care of the acutely ill hospitalized patient," Critical Care, vol. 8, pp. 33-40, 2004.

[36] P. Gualdiero, "Clinical features, anthropometric characteristics, and racial influences on the white coat effect in a single centre cohort of 1553 consecutive subjects undergoing routine ambulatory blood pressure monitoring," Blood Press Monit, vol. 5, pp. 53-57, 2000. 